\title{
Characterization of Complex Crude Oil Microemulsions-DSC Contribution
}

\author{
Ayako Fukumoto, Christine Dalmazzone", Didier Frot, Loïc Barré and Christine Noïk \\ IFP Energies nouvelles, 1-4 avenue de Bois Préau, 92852 Rueil-Malmaison - France \\ e-mail: christine.dalmazzone@ifpen.fr \\ *Corresponding author
}

\begin{abstract}
Surfactant flooding is a chemical enhanced oil recovery (EOR) process which consists in injecting optimized formulations of surfactants in the reservoir in order to remobilize the residual oil trapped in the pores of the rock. To do that, it is necessary to design specific formulations in order to get so-called Winsor III systems of very low interfacial tensions with the crude oil. Unfortunately, there is no well-established way to characterize and understand the physical properties and structures of microemulsions composed of crude oil and industrial surfactants due to their extreme complexity. In a previous work, we have developed a methodology based on the use of several techniques (DLS, MLS, SAXS, cryo-SEM, DSC, interfacial measurements, etc.) allowing physico-chemical and morphological characterization of these microemulsions in the case of a model system. In this article, we will demonstrate how DSC can be used to provide information on the physico-chemical composition of complex microemulsions (water and oil content, salinity, etc.) and on their morphology (continuous phase, dispersed phase, etc.).
\end{abstract}

\section{INTRODUCTION}

Surfactant flooding is a chemical enhanced oil recovery (EOR) process in which the key mechanism is to reduce interfacial tension (IFT) between oil and the displacing aqueous formulation of surfactants. Chemical EOR operations consist of a combination of surfactant and polymer or alkaline, surfactant, and polymer or any variation of these main injection processes [1]. As a matter of fact, an optimum formulation of surfactants has to be injected in the reservoir in order to remobilize the oil trapped in the pores of the reservoir rock. This so-called optimum formulation corresponds to a minimum IFT and a maximum in oil recovery $[2,3]$. It is generally obtained for an appropriate balance of the hydrophobic and hydrophilic affinities of the surfactants mixture for the water and oil phases, corresponding to Winsor III microemulsions systems [2]. Microemulsions are thermodynamically stable systems composed of water, oil, surfactants and sometimes co-surfactants such as alcohols
[4]. Generally, microemulsions are classified according to a typology proposed in 1948 by Winsor [5]. One can observe the structural transition of microemulsions from Winsor I to Winsor II through Winsor III by changing physical parameters of a system like temperature or salinity. In the case of EOR processes, it is generally performed through salinity scans due to the anionic nature of the surfactants used [1]. The Winsor I type is a biphasic system in which the supernatant organic phase is in equilibrium with an $\mathrm{O} / \mathrm{W}$ microemulsion where the surfactant is predominantly present [6]. The Winsor II type also corresponds to a biphasic system, where a W/O microemulsion in which most of the surfactant is found is in equilibrium with the aqueous phase. The Winsor III system designates a three-phase system in which a surfactant-rich intermediate phase forms between the aqueous and organic phases. This microemulsion consists generally of a bicontinuous system of aqueous and organic phases, without micelles or predominant continuous phase. A Winsor IV system may also be 
encountered, which in fact corresponds to a Winsor I, II or III, without excess of aqueous phase or organic phase (monophasic microemulsion system).

Even if microemulsions have been studied for different industrial applications [7-10], there is no well-established methodology to characterize and describe the physical properties and morphologies of microemulsions composed of crude oil and industrial surfactants in the case of chemical EOR, due to their utmost complexity. That is the reason why, referring to various works performed on microemulsions made of model oils and surfactants [11-18], we have developed an experimental methodology based on the application of several probes, i.e. DLS (Dynamic Light Scattering), MLS (Multiple Light Scattering), SAXS (Small Angle X-ray Scattering), cryo-SEM (Scanning Electron Microscopy by cryo-fracture), DSC (Differential Scanning Calorimetry), interfacial measurements, etc. [19]. This methodology was validated in the case of a model microemulsion system made of SDBS (Sodium Dodecyl Benzenesulfonate), Isobutanol, Brine, and Decane. The objective was to be able to address the case of more complex systems such as the one including real oils and formulations of surfactants, which is of prime importance to chemical EOR, especially surfactant flooding. Among the different techniques that can be applied to investigate complex dispersed systems as emulsions or microemulsions, DSC appears as a technique that can be used for physico-chemical characterization as well as for morphological studies [17,19-24]. In this paper, we will show how DSC can be advantageously used in addition to other classical techniques to investigate complex microemulsions made of real crude oil and representative EOR formulations of surfactants.

\section{MATERIALS AND METHODS}

\subsection{Materials}

Dodecane (99\% purity) was purchased from VWR chemicals. The industrial surfactants were provided by Solvay. All compounds were used without further purification. The so-called AI system was composed of alkylglyceryl ether sulfonate, AGES (33\% active matter) and Internal Olefin Sulfonate, IOS (33\% active matter). Aqueous solutions were prepared with Milli-Q water made with a resistivity of $18.2 \mathrm{M} \Omega / \mathrm{cm}$. The crude oil AL comes from the Middle-East and was chosen in this study because it is easy to handle at room temperature. It was filtered through a filter of $5 \mu \mathrm{m}$. Details of the crude composition are listed in Table 1 . Here, the composition was determined by the socalled SARA analysis performed by the Analysis department in IFPEN. This analysis is based on IP-143 for the determination of asphaltenes (insoluble in $\mathrm{nC7}$ ) and ASTM D-2007 for the determination of saturates, aromatics and
TABLE 1

Density, $\mathrm{API}^{\circ}$ and viscosity at $20^{\circ} \mathrm{C}$ and SARA composition of crude oil $\mathrm{AL}$.

\begin{tabular}{l|l}
\hline Density, $\mathrm{g} / \mathrm{cm}^{3}$ & 0.88 \\
\hline API degree, ${ }^{\circ}$ & 29 \\
\hline Viscosity, $\mathrm{mPa} \cdot \mathrm{s}$ & 12 \\
\hline Saturates, $\mathrm{wt} \%$ & 53.8 \\
\hline Aromatics, wt $\%$ & 32.6 \\
\hline Resins, wt $\%$ & 10.6 \\
\hline Asphaltenes, wt $\%$ & 2.7 \\
\hline
\end{tabular}

TABLE 2

Summary of compositions of all the systems studied in this work

\begin{tabular}{l|l|l|l}
\hline Surfactant and concentration & Oil & Name & $\mathrm{NaCl}, \mathrm{g} / \mathrm{L}$ \\
\hline AGES $10 \mathrm{~g} / \mathrm{L}+\mathrm{IOS} 10 \mathrm{~g} / \mathrm{L}$ & Dodecane & AI-C12 & $80-100$ \\
\hline AGES $10 \mathrm{~g} / \mathrm{L}+\mathrm{IOS} 10 \mathrm{~g} / \mathrm{L}$ & Crude oil AL & AI-AL & $30-130$ \\
\hline
\end{tabular}

resins. It is noted that salinity scans performed have shown that the average Equivalent Alkane Carbon Number (EACN) of AL crude oil is around 12 (which is equivalent to dodecane).

\subsection{Preparation of Samples}

Microemulsions were prepared at room temperature $\left(20 \pm 2{ }^{\circ} \mathrm{C}\right)$ in a glass tube by the following procedures. First, mother solutions of AGES $(40 \mathrm{~g} / \mathrm{L})$, IOS $(40 \mathrm{~g} / \mathrm{L})$, and $\mathrm{NaCl}$ $(200 \mathrm{~g} / \mathrm{L}$ for AI system) were prepared separately in flasks. The mass of the surfactants and $\mathrm{NaCl}$ was measured with an electronic mass balance (AE200, Mettler Toledo) with a precision of $\pm 0.1 \mathrm{mg}$. Second, for the AI system, $2.5 \mathrm{ml}$ of the AGES and IOS solutions were poured, respectively. After, water and $\mathrm{NaCl}$ solution were poured in the glass tube with pipettes. Here, the volume of water and the $\mathrm{NaCl}$ solution was calculated from the set salinity (studied range is listed in Tab. 2) and set volume of aqueous phase (10 $\mathrm{ml}$ for AI system). The solution was carefully mixed. Finally, oil, of which volume was the same as the aqueous phase (water to oil ratio, WOR $=1$ ), was put in the glass tube. Samples were again mixed gently by turning the tubes upside down for approximately 20 times, and left to be equilibrated for 1 month at room temperature. The compositions of each system are summarized in Table 2 . The concentration listed in the table is that of the aqueous phase.

For the analyses performed on the middle phase microemulsion (Winsor III system), an intermediate phase sample was taken very carefully using a syringe and a 
needle. Initially, the needle is filled with air to eliminate contamination by passing through the supernatant oil phase. Once the needle is placed in the middle of the intermediate phase, the air is gently expelled and a small part of this phase is removed. The needle is then disconnected from the syringe and a new needle is used to transfer the sample without risk of contamination with the supernatant oil phase.

\subsection{Spinning Drop Method}

The interfacial tension (IFT) of microemulsion and aqueous/ oil phase was measured at $20^{\circ} \mathrm{C}$ using the spinning drop video tensiometer (SVT 20N, Data Physics). Equilibrated solutions were sampled in the case of AI-C12 system for the Winsor III microemulsion. First, density of the sample was measured with a densimeter (DMA $4500 \mathrm{M}$, Anton Paar) at $20^{\circ} \mathrm{C}$. After, about $1 \mathrm{ml}$ of the aqueous phase was inserted in a glass capillary, and a drop of the middle phase, contacting with the aqueous phase, was injected. The capillary was rotated at high speed $(5000 \mathrm{rpm})$. When the drop reaches an equilibrium shape, IFT can be calculated with the balanced centrifugal force as $\Upsilon=\omega^{2} \cdot r^{3} \Delta \rho / 4$ [25]. Here, $\Upsilon$ is IFT, $\omega$ is angular velocity, $r$ is drop radius, and $\Delta \rho$ is density difference between the phases .

It is noted that for AI-AL system, IFTs were measured from non-equilibrated aqueous and oil phases on a large range of salinities. In either way, the IFT was measured until it reached a constant value for at least ten minutes to be sure that it was obtained at the equilibrated state.

\subsection{Karl-Fischer Titration}

The water content in the middle phase microemulsion was measured by the Karl-Fischer titration. This titration was made with a 787-KF Titrino from Metrohm with a reagent of HYDRANAL ${ }^{\circledR}$-Composite 5 purchased from Fluka. A sample of about $10 \mathrm{mg}$ was precisely weighted and injected into a measuring cell, and the amount of water in the sample was calculated according to the volume of reagent consumed. All the samples were analyzed at least three times.

\subsection{Hyamine Titration}

Concentration of surfactants in the aqueous phase was determined by the Hyamine titration (Hyamine 1622 solution, Merck Millipore) using a titrator (862-compact titrosampler, Metrohm). $1 \mathrm{ml}$ of the sample was inserted in the measuring cell. The titrant (Hyamine) was added in the sample with an increment of $0.03 \mathrm{ml}$. An electrode was used for the potentiometric titration to determine the amount of anionic surfactants in the sample.

\subsection{Multiple Light Scattering (MLS)}

MLS can be used to determine precisely the different interfaces involved with complex microemulsions, especially with crude oil. We used a specific apparatus called Turbiscan MA 1000, developed by the French Company FormulAction. It is an automated, vertical scan macroscopic analyzer that is used to study the stability of concentrated colloidal solutions (emulsions, suspensions, foams, etc.). It works with a near infrared light source. The vertical scanning of the sample is combined with a double detection system: transmission and light scattering (backscattering). It carries out step-by-step vertical scanning of the entire sample in a few seconds. The intensity of transmitted light is used for clear and slightly turbid systems and back-scattered light is useful for studying opaque and very turbid systems.

\subsection{Cryo-SEM/EDS}

The cryo-SEM technique was used to visualize the structure of the middle phase microemulsions. In this study, a small piece of the middle phase at the optimal salinity was shock frozen in nitrogen slush at $-210^{\circ} \mathrm{C}$, fractured, metallized with chromium and transferred in a vacuum to the scanning electron microscope (Supra 40, Zeiss) fitted with a cold stage unit. An EDS analysis (energy dispersive X-ray spectroscopy) was used to determine the main elements $(\mathrm{C}, \mathrm{Cl}, \mathrm{Na}, \mathrm{S}$, $\mathrm{O}$, etc.).

\subsection{DSC}

Crystallization and dissociation temperatures/enthalpies of middle phase microemulsions were measured using a DSC apparatus (DSC 1, Mettler Toledo). The calibration was performed with high purity indium. Water was used to determine the uncertainty of the melting temperature and enthalpy, which were found to be $0.40^{\circ} \mathrm{C}$ and $20 \%$, respectively. The uncertainty on enthalpy appears as quite high, but it could be decreased by completing the calibration with materials showing a melting temperature below $0{ }^{\circ} \mathrm{C}$, as mercury for instance. Between 10 and $20 \mathrm{mg}$ of middle phase microemulsions were inserted in a the DSC crucible. The temperature of the furnace was decreased from 20 to $-80^{\circ} \mathrm{C}$ at a cooling rate of $5^{\circ} \mathrm{C} \cdot \mathrm{min}^{-1}$, and kept constant for $5 \mathrm{~min}$. Then, the temperature was increased from -80 to $20^{\circ} \mathrm{C}$ at a heating rate of $5^{\circ} \mathrm{C} \cdot \mathrm{min}^{-1}$. The temperatures of crystallization and melting as well as the melting enthalpy were measured to investigate the water morphology and salinity of the microemulsions. This scanning analysis was also performed with the solutions of AI surfactants as a reference. 


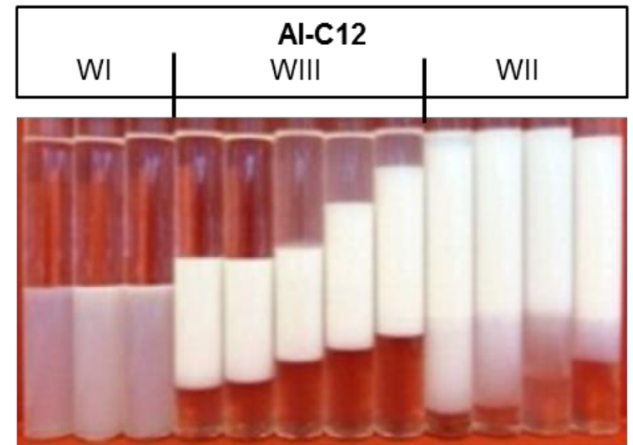

$\begin{array}{llllllllllll}1 & 2 & 3 & 4 & 5 & 6 & 7 & 8 & 9 & 10 & 11 & 12\end{array}$

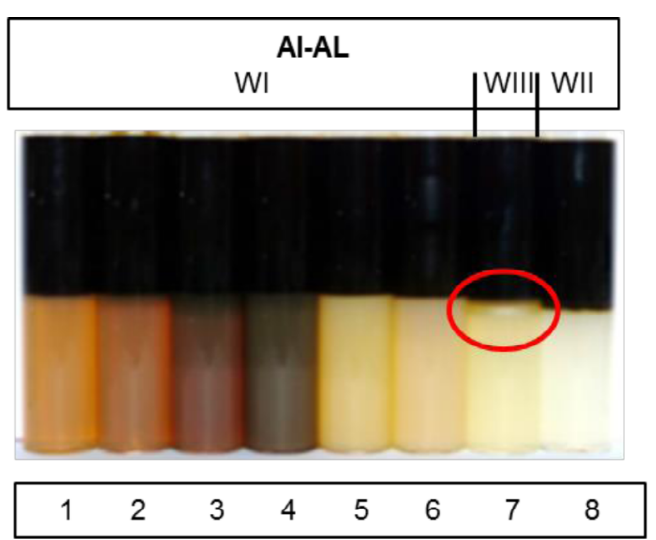

Figure 1

Salinity scans with AI-C12 (left) and AI-AL (right) systems-AI-C12: Tube 1: $80 \mathrm{~g} / \mathrm{L}$, Tubes 2 to $12: 90$ to $110 \mathrm{~g} / \mathrm{L}$ (interval of salinity of $2 \mathrm{~g} / \mathrm{L}$ )The middle phase (WIII) is opaque (white) ; AI-AL: Tubes 1 to $8: 30$ to $100 \mathrm{~g} / \mathrm{L}$ (interval of salinity of $10 \mathrm{~g} / \mathrm{L}$ )-The middle phase (WIII) surrounded in red is opaque (yellow).
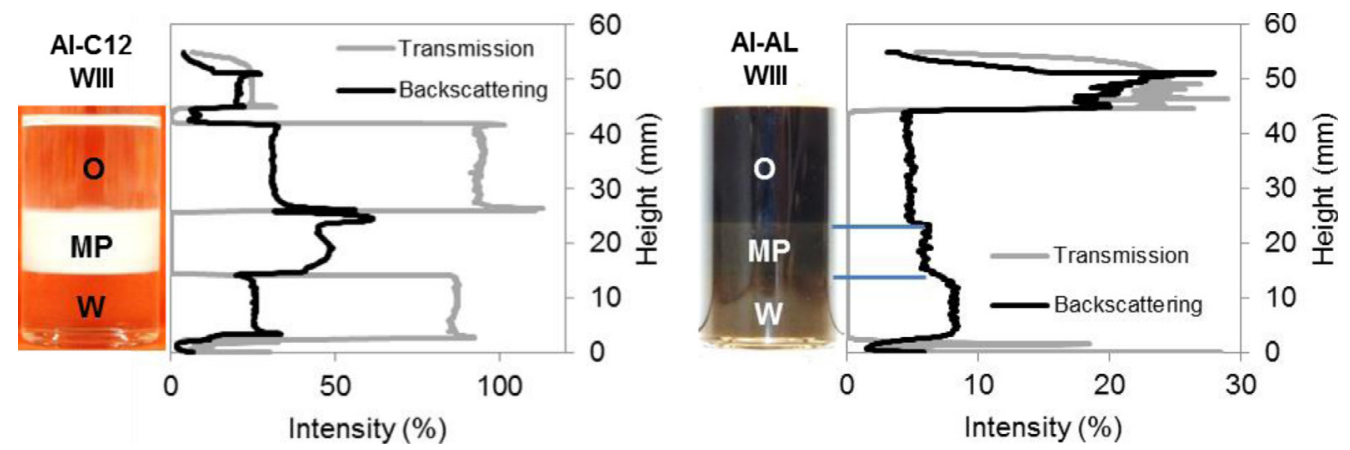

Figure 2

MLS analysis of Winsor III systems (Left: AI-C12; Right: AI-AL)-O: oil phase in excess-MP: middle phase-W: water phase in excess.

\section{RESULTS AND DISCUSSION}

\subsection{Physicochemical Analysis}

\subsubsection{Phase Behavior}

The phase transitions of the samples were investigated by changing salinities. All the samples were kept at room temperature for 1 month in order to have equilibrated phases (the equilibrium is considered to be reached when no evolution of the phases volumes is observed). Figure 1 shows a summary of the salinity scans performed. White-opaque middle phases (WIII) were observed from 94 to $102 \mathrm{~g} / \mathrm{L}$ for AI-C12 system (optimal salinity at $98 \mathrm{~g} / \mathrm{L}$ ). As for AI-AL system, a middle phase emerged only at $90 \mathrm{~g} / \mathrm{L}$. This middle phase was yellowcolored and the volume was very small. It is true that classical microemulsions are expected to be transparent. But with crude oils and formulation of surfactants specifically designed for chemical EOR, this is generally not the case. Nevertheless, we are sure that these systems are not separated emulsions phases because they form spontaneously (no agitation is provided during the salinity scans) and the IFT is ultra-low (as will be shown in Sect. 2.1.3).

\subsubsection{Multiple Light Scattering}

Interfaces of the Winsor III system at the optimum salinity were determined by Turbiscan ${ }^{\mathrm{TM}}$. Figure 2 shows the results of the samples composed of model oil (AI-C12). In the case of model oil, the position of the middle phase is easily determined by sight because excess aqueous and oil phases are highly transparent. It can be seen in the intensity of the transmission signal. It is clear that interfaces can be determined by the intensity of both the transmission and the back scattering signals.

The sample composed of crude oil was also analyzed. The results are shown in Figure 2 (right). For AI-AL sample, the concentration of surfactants was increased to 5 times as written in Table 2 in order to have a large volume of the middle phase, as expected from a theoretical point of view. The interfaces were difficult to determine with a visual observation because all the phases were dark-colored as can be seen in the picture of the sample in Figure 2. Indeed, the intensity of the transmission was zero through all the phases. The measured intensity in the back scattering showed the slight differences between the three phases, and this enabled 
TABLE 3

Summary of IFT and optimum salinity .

\begin{tabular}{l|l|l}
\hline System & AI-C12 & AI-AL \\
\hline Optimum salinity, g/L & $\begin{array}{l}98 \\
(99)\end{array}$ & $\begin{array}{l}90 \\
(99)\end{array}$ \\
\hline IFT, mN/m & $\begin{array}{r}2.4 \times 10^{-3} \\
\left(1.0 \times 10^{-3}\right)\end{array}$ & $\begin{array}{r}2.5 \times 10^{-3} \\
\left(1.7 \times 10^{-2}\right)\end{array}$ \\
\hline
\end{tabular}

${ }^{a}$ value in a parenthesis is reported in literature [26]

us to determine the position of the interfaces. It is shown that this analysis by MLS is powerful in determining precisely the position of interfaces when the system exhibits dark phases such as crude oil.

\subsubsection{IFT}

IFTs between microemulsion and oil/water phases were measured with the spinning drop method. Here, equilibrated phases were used for AI-C12 system at the optimal salinity (one drop of the middle phase was injected in the capillary filled with the water phase in excess), because the measurement is easier by this way. For the AI-AL system, for which the volume of the middle phase was too small, non-equilibrated aqueous and oil phases were used in a wide range of salinities. In either way, the IFT was measured until it reached a constant value for at least ten minutes to be sure that it was obtained at the equilibrated state. A summary of the IFT at the optimum salinity is shown in Table 3. Figure 3 shows the IFTs of AI systems, respectively. While IFTs were measured in a wide range of salinities for AI-AL system, only one IFT at a salinity of $98 \mathrm{~g} / \mathrm{L}$ was measured for AI-C12 system. The lowest IFT was found as expected at $90 \mathrm{~g} / \mathrm{L}$ for AI-AL system, which is in the same order of magnitude as AI-C12 system.

\subsubsection{Water Content of Oil Phase and Surfactant Concentration in Aqueous Phase}

Some measurements were performed to confirm the optimal salinity of the crude oil system (AI-AL), for which it was difficult to identify the middle phase corresponding to Winsor III by a simple mass balance (because the different phases are difficult to visualize), contrary to the dodecane system where it can be done easily. The compositions of the excess oil and aqueous phases were investigated by the Karl-Fischer method and the Hyamine titration in the case of this AI-AL system. These titration methods allow a precise determination of the water and surfactant partition in the different phases during the salinity scan, which is not possible with the mass balance from visual observation. Figure 4 shows that the water content in the oil phase increases gradually along with the salinity increment. The concentration of the surfactant in the water phase decreases rapidly at a salinity of $100 \mathrm{~g} / \mathrm{L}$, however still a

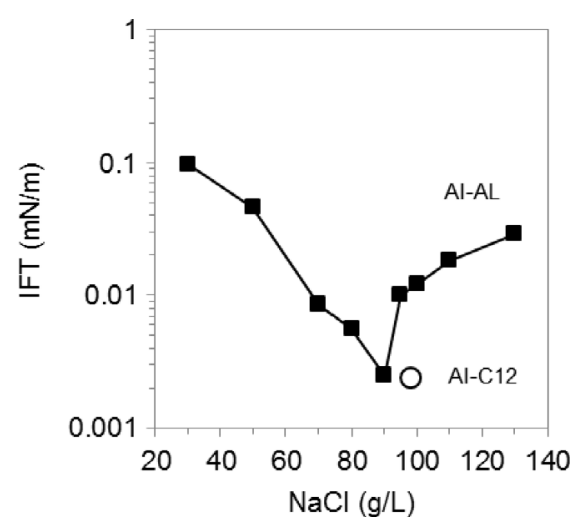

Figure 3

IFTs of AI systems. O is AI-C12 and $\square$ is AI-AL (note that the $\mathrm{y}$ axis is in logarithmic scale).

significant (30\%) amount remains in the aqueous phase. It is noteworthy that both curves cross around $90 \mathrm{~g} / \mathrm{L}$, which corresponds to the salinity that was determined by the salinity scans. This result is consistent with what was expected: in the Winsor I region (low salinity), most of the surfactant is found in the aqueous phase, while it is gradually transferred to the middle phase (Winsor III) and the oil phase (Winsor II) as the salinity increases. On the other hand, if we consider the water content in the oil phase, this value is relatively low for Winsor I and tends to increase as the salinity increases due to the formation of a w/o microemulsion.

\subsubsection{Salinity and Water Content of Microemulsion}

From the heat profiles of the melting process obtained from DSC measurements, one can estimate the concentration of $\mathrm{NaCl}$ in the samples of microemulsions by comparing the temperature corresponding to the end of the progressive melting of ice with that of the aqueous solutions. On the other hand, water content of microemulsion can also be estimated by comparing the total enthalpies (surface area of the peaks) of the eutectic and progressive melting of ice in microemulsions with that in aqueous solutions [23].

So to get access to the salinity and water content of microemulsions samples, it is first necessary to analyze the thermogrammes of the aqueous phases and oil phases separately as references. Figure 5 shows heat profiles of AI solutions at different salinities and dodecane. During the melting process of the aqueous solutions of surfactants with $\mathrm{NaCl}$, two endothermic peaks were observed; the first one detected around $-21{ }^{\circ} \mathrm{C}$ corresponds to the eutectic melting of $\mathrm{NaCl}$ and ice, and the second broad peak detected soon after the first one, can be attributed to the progressive melting of ice. Here, the temperature of progressive melting of ice was extrapolated from an intersection point of a baseline and an inclined line which goes through the apex of the progressive melting peak. The slope of this line corresponds to that of the tangent at the inflection point of the eutectic melting peak. A 


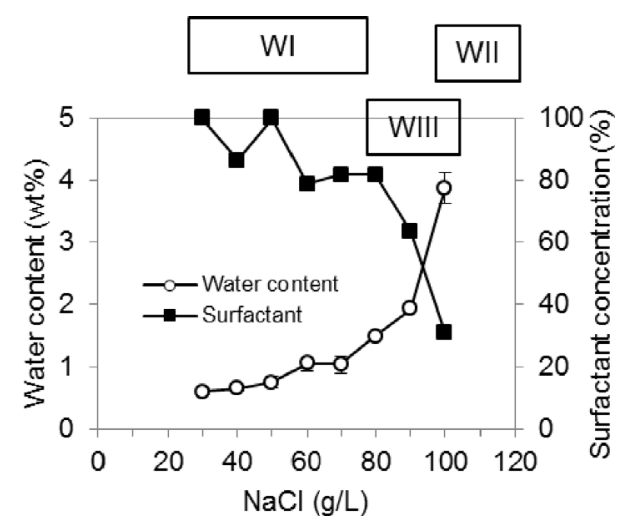

Figure 4

Water content of oil phase and concentration of surfactant in aqueous phase for AI-AL. Measurements were conducted with Karl-Fischer and Hyamine titration.

relation between the temperature of the end of progressive melting of ice and salinity was found as $T=-0.11 x+4.98$ with AAD of $0.02{ }^{\circ} \mathrm{C}$, where $T$ is the melting temperature in ${ }^{\circ} \mathrm{C}$ and $x$ is the concentration of $\mathrm{NaCl}$ in $\mathrm{g} / \mathrm{L}$. It is noted that this relation is only valid within the studied salinities and cannot be extrapolated. This relation will be used to determine the salinity of the water phase knowing the temperature of the end of melting of the aqueous phase contained in the middle phase microemulsion. It seems that the effect of the anionic surfactants on the $\mathrm{NaCl}$-water phase diagram is not significant (the melting temperatures are of the same order of magnitude). A relation between the melting enthalpy and salinity, when water content was $100 \%$, was found as $\Delta H=2.03 x-5.07$ $\times 10^{2}$ with the ARD of $1.4 \%$ (within the studied range of salinities). This relation will be used to determine the water content of the middle phase knowing the enthalpy of melting determined by integration from the thermogramme. It is noteworthy that the melting peak of dodecane is superimposed with the peak corresponding to the progressive melting of ice. The melting temperature of dodecane, determined by the onset temperature, is $-9.7^{\circ} \mathrm{C}$, which corresponds to the literature data. Concerning the crude oil AL, no peak was observed in the range of temperatures that we consider (Fig. 6).

Heat profiles of AI-C12 middle phase microemulsion at a salinity of $98 \mathrm{~g} / \mathrm{L}$ are shown in Figure 7 (WIII). Three peaks were observed during the melting process. The first one at $-21{ }^{\circ} \mathrm{C}$ is due to the eutectic melting. The second and third one are attributed to dodecane and the progressive melting of ice, respectively. Since the second and the third peaks are overlapping each other, the water content in the microemulsion was not determined. But it is not really an issue because this value can be easily deduced from the analysis of the phases volumes from the salinity scan $(64.5 \mathrm{v} \%$, i.e. around $72 \mathrm{wt} \%$ ). It was possible to estimate a value of the salinity by determining the temperature of the end of melting of ice: around $90 \mathrm{~g} / \mathrm{L}$ (slightly lower than the

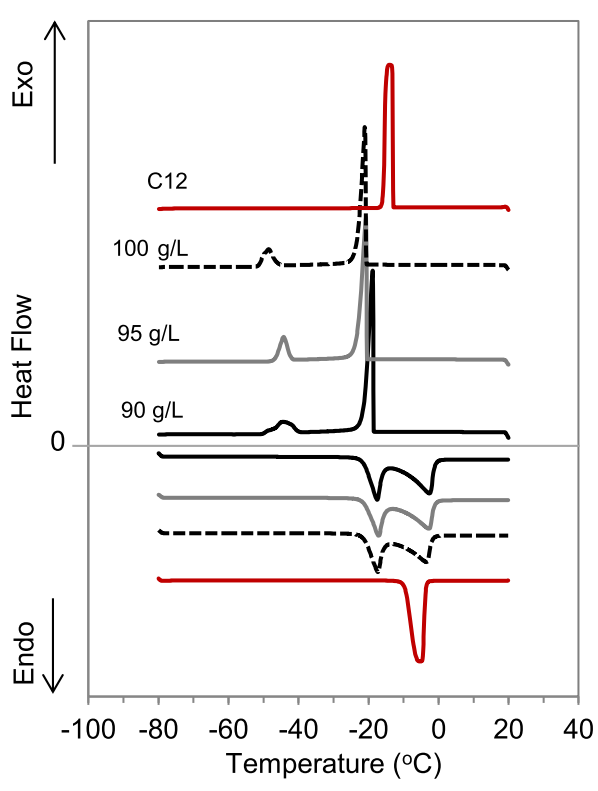

Figure 5

Heat flow profiles of AI solutions at different salinities and dodecane.

expected salinity of $98 \mathrm{~g} / \mathrm{L}$ ). The same tendency was observed in the case of the previously studied model system [19].

The heat profile of AI-AL middle phase microemulsion is also shown in Figure 7. The eutectic and progressive melting of ice was detected during the heating process. Note that the $\mathrm{AL}$ crude oil has no contribution to the peak as shown in Figure 6. The concentration of $\mathrm{NaCl}$ and water content of microemulsion estimated from the DSC measurement are $109 \mathrm{~g} / \mathrm{L}( \pm 4)$ and $80.1 \mathrm{wt} \%( \pm 16)$ respectively. The salinity value appears as significantly higher than the expected salinity $(90 \mathrm{~g} / \mathrm{L})$. An hypothesis is that it could be attributed to the very high concentration of anionic surfactants in the middle phase of the microemulsion. The water content determined by DSC was found higher than the value measured from the Karl Fisher titration $(64.4 \mathrm{wt} \% \pm 1$, i.e. around $56 \mathrm{v} \%$ ). But if we take into account that the uncertainty is quite large with DSC due to the small amount of sample required for the analysis (a few mg against $10 \mathrm{mg}$ for Karl Fischer), the lower value (64.1 wt \%) of the DSC test corresponds to the average value of the titration. So the results are consistent between both methods.

\subsection{Morphological Analysis}

\subsubsection{Images Of Middle Phase Winsor III Microemulsions}

Cryo-SEM allowed us to get the pictures of the structure of the middle phase microemulsions with both systems. Figure 8 shows the images of the AI-C12 middle phase microemulsion at a salinity of $98 \mathrm{~g} / \mathrm{L}$. An interesting point is that this phase is different from the classical bicontinuous phase which is 


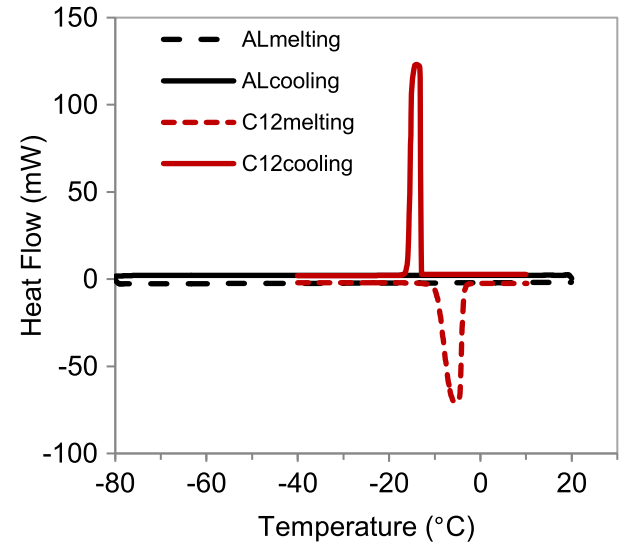

Figure 6

Heat flow profiles of dodecane and crude oil AL.

generally found in the literature on model systems [16,19, 27] but is rather constructed as a brick wall; one phase is surrounded by a layer of another phase, and they seem to make an array. Figure 8 (right) shows a more detailed picture in comparison to (left). From the figure, one can see that the brick is a smooth phase and the mortar layer, which is brighter, is composed of small substances. An EDS analysis has shown that the smooth phase was essentially composed of water (with some traces of $\mathrm{Na}, \mathrm{Cl}$ and $\mathrm{C}$ ), while the mortar layer is made of grains between 40 and $250 \mathrm{~nm}$, mainly composed of C, O, Na, $\mathrm{Cl}$ and $\mathrm{S}$ traces (i.e. oil, water, salt and surfactant).

Note that Moiré reported the image of the microemulsion of AI-C12 at a salinity of $99 \mathrm{~g} / \mathrm{L}$, which shows a similar structure and similar conclusions regarding the EDS analysis [26].

The middle phase microemulsion of AI-AL at a salinity of $90 \mathrm{~g} / \mathrm{L}$ is shown in Figure 9. It appears that the structure is similar to the one of AI-C12, which has a brick-wall structure. The EDS analysis clearly demonstrates that the smooth areas are essentially made of water and some traces of $\mathrm{Na}, \mathrm{Cl}$ and $\mathrm{C}$. The mortar layers, which appear brighter, are made of grains between $100 \mathrm{~nm}$ and $1 \mu \mathrm{m}$, which composition revealed by EDS analysis is mainly $\mathrm{C}, \mathrm{O}, \mathrm{Na}, \mathrm{Cl}$ and some traces of $\mathrm{S}$ (i.e. oil, water, salt and surfactant, as in the previous case).

\subsubsection{Crystallization of Microemulsions}

Dalmazzone et al. [23] demonstrated the characterization of opaque-emulsions using a DSC. Temperature of crystallization is related to morphology of water in emulsions since a probability of nucleation of ice is dependent of the volume of water. It has been shown in the literature that the smaller the volume is, the lower the freezing temperature is [20].

The thermogrammes of crystallization of the AI-C12 middle phase microemulsion are shown in Figure 10 for two close salinities, i.e. $98 \mathrm{~g} / \mathrm{L}$, which corresponds to the optimal salinity according to the salinity scan, and $99 \mathrm{~g} / \mathrm{L}$, which

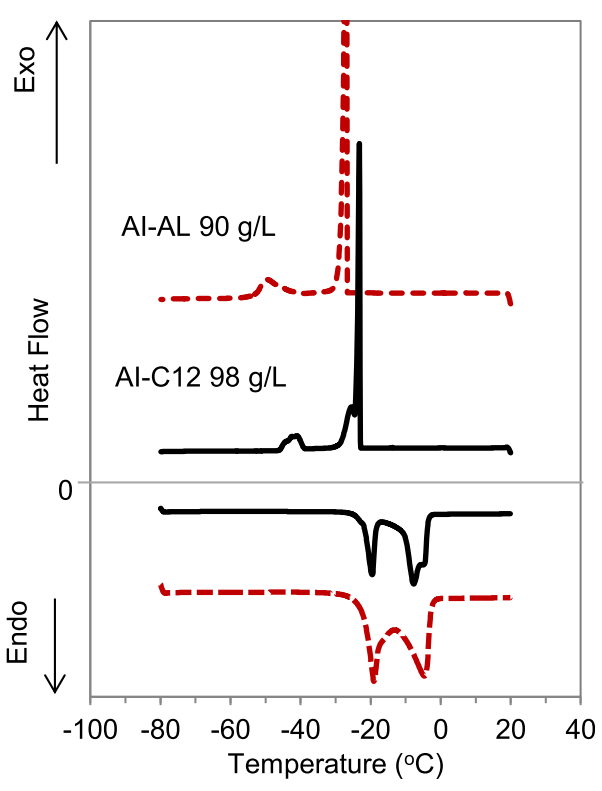

Figure 7

Heat flow profiles of AI-C12 WIII middle phase microemulsion $(98 \mathrm{~g} / \mathrm{L}$ of $\mathrm{NaCl})$ and $\mathrm{AI}-\mathrm{AL}$ WIII middle phase microemulsion $(90 \mathrm{~g} / \mathrm{L}$ of $\mathrm{NaCl})$.

corresponds to a Winsor $\mathrm{III}^{+}$system (which tends towards a Winsor II, i.e. w/o microemulsion). During the cooling process, a sharp positive peak was observed around $-22^{\circ} \mathrm{C}$ with the microemulsion at a salinity of $98 \mathrm{~g} / \mathrm{L}$. The second peak started around $-25^{\circ} \mathrm{C}$, which was before the first one came back to the base line. The temperature of the two peaks are very close and the peaks can almost be considered as one sharp peak with a wide base. This leads to an observation that in the microemulsion with the brick-wall structure at the optimum salinity, the crystallization of oil was induced by that of neighboring water. The third peak detected around $-40{ }^{\circ} \mathrm{C}$ is attributed to $\mathrm{NaCl}$ crystallization. Interestingly, the first two peaks were not overlapping each other with the microemulsion at a salinity of $99 \mathrm{~g} / \mathrm{L}$. This indicates that an increment of $1 \mathrm{~g} / \mathrm{L}$ of $\mathrm{NaCl}$ seems to have a significant effect on the morphology of the microemulsion. But this experiment should be repeated many times to confirm it due to the random character of nucleation. So the cryo-SEM and DSC analyses are consistent because cryo-SEM shows the presence of large domains of water that tend to crystallize rapidly, without a significant amount of supercooling, which would be the case if the water was finely dispersed. Furthermore, the fact that dodecane crystallizes after water suggests that the dodecane is finely divided, because the temperature of crystallization shows a significant supercooling (difference between the melting and crystallization temperatures), between 11 and $16^{\circ} \mathrm{C}$, compared to bulk dodecane for which the supercooling is around $3{ }^{\circ} \mathrm{C}$ (see Fig. 10). Note that many studies have reported significant supercooling degrees with emulsified normal alkanes [22]. 


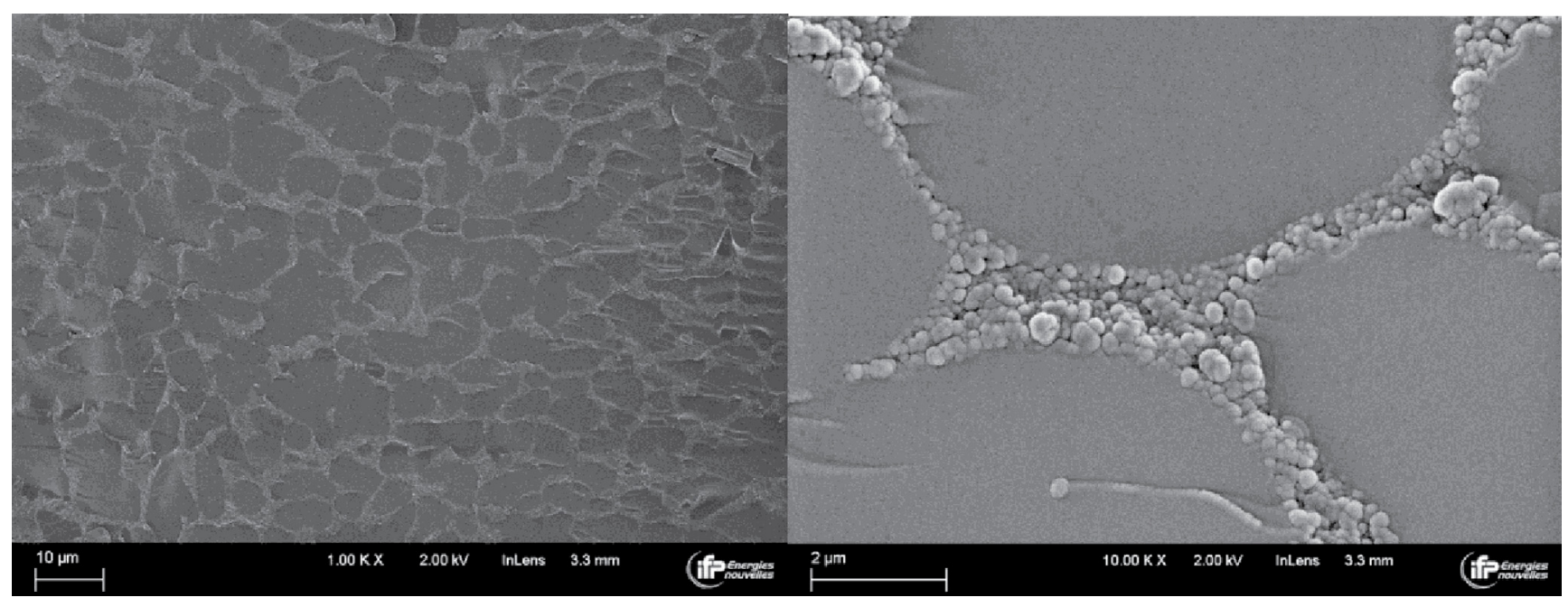

Figure 8

Cryo-SEM pictures of the middle phase microemulsion at a salinity of $98 \mathrm{~g} / \mathrm{L}$ with the AI-C12 system (left: general view; right: focus on the mortar layer).

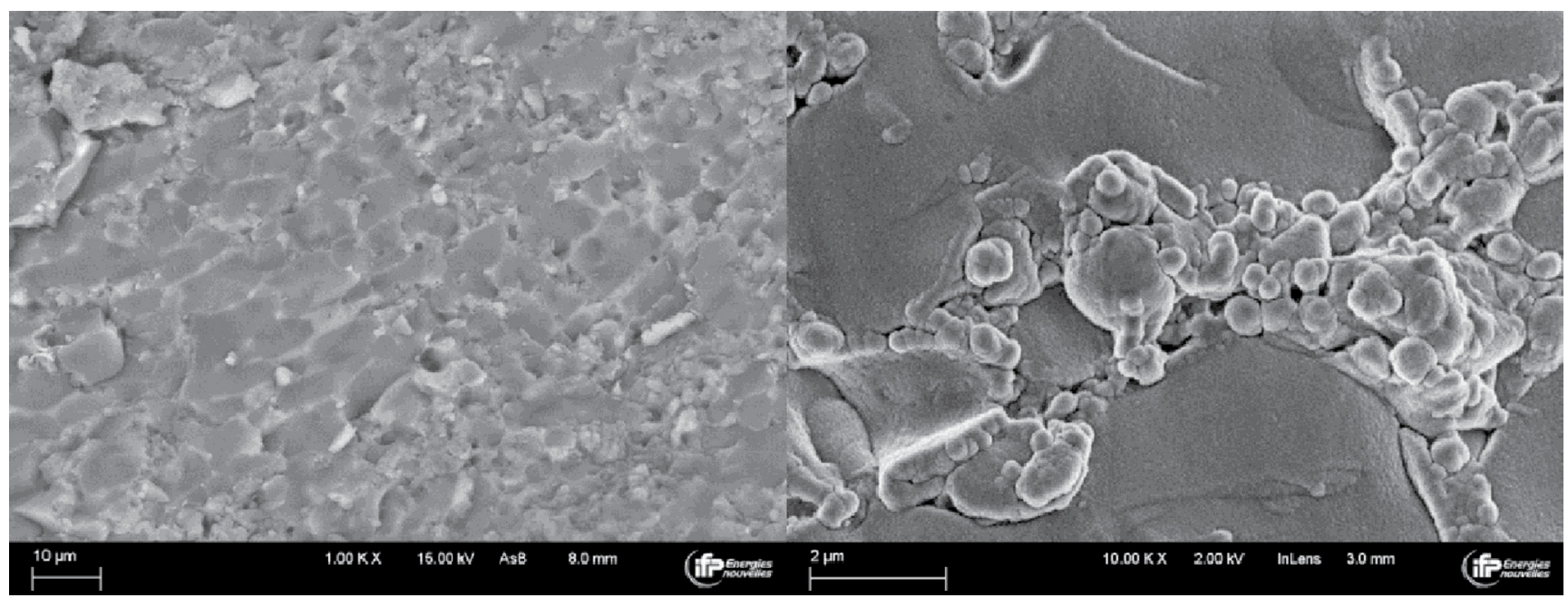

Figure 9

Cryo-SEM pictures of the middle phase microemulsion at a salinity of $90 \mathrm{~g} / \mathrm{L}$ with the AI-AL system (left: general view; right: focus on the mortar layer).

Of course, we cannot rule out the hypothesis that cooling provokes the separation of the system during the DSC test, explaining the sharp peak of crystallization of water. But in that case, dodecane should also crystallize at a temperature close to the one of bulk dodecane, which is not the case.

The thermogrammes of crystallization of the middle phase microemulsion of AI-AL system at a salinity of $90 \mathrm{~g} /$ $\mathrm{L}$ were previously presented in Figure 7 (Sect. 2.1.5). Two positive peaks were observed during the cooling process. The first one is at $-26^{\circ} \mathrm{C}$, it is very abrupt and sharp, characteristic of the crystallization of bulk water. The second peak observed at $-50{ }^{\circ} \mathrm{C}$ corresponds to the $\mathrm{NaCl}$ crystallization. Note that there is around $10^{\circ} \mathrm{C}$ of difference in the crystallization signal attributed to $\mathrm{NaCl}$ between $\mathrm{AI}-$
C12 and AI-AL systems, which seems quite a lot even if the crystallization temperature is not an invariant. It is worth mentioning that the crystallization peak of water is about $8^{\circ}$ lower than the one detected with the bulk aqueous solution at the same salinity (Fig. 5). This significant supercooling could indicate that the water channels are not very well connected. Unfortunately, as the crude oil shows no peak in the heat profile (Fig. 6), it is not possible to conclude on the state of dispersion of the oil in the microemulsion. Nevertheless, if we exclude the signals corresponding to oil, one can say that the crystallization thermogrammes of AI-C12 and AI-AL are very similar, which suggests that the distribution of water is nearly the same for both systems. This is confirmed by the cryo-SEM analysis that shows a 


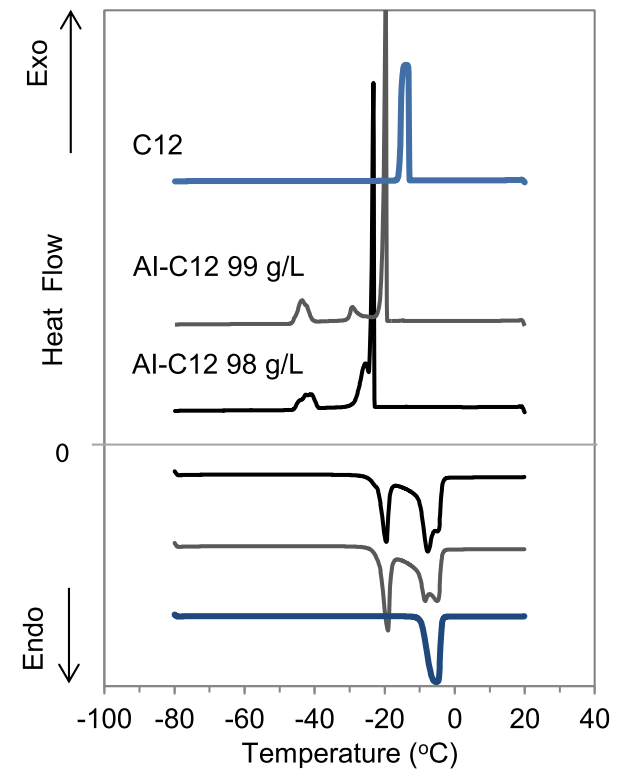

Figure 10

Heat flow profiles of AI-C12 middle phase microemulsions and bulk dodecane.

brick-wall structure with large domains of water (Fig. 9). So once again, the cryo-SEM and DSC analyses are consistent.

Note that in the case of previously studied model microemulsions which exhibited a bicontinuous structure near the optimum salinity, as revealed by cryo-SEM analysis, the DSC heat profiles showed a simultaneous crystallization of water and oil [19]. With the present complex systems, AI-C12 (complex surfactants-model oil) and AI-AL (complex surfactants-complex crude oil), the structure is clearly different from the bicontinuous one.

\section{CONCLUSION}

In this study, we have investigated the physicochemical properties as well as the morphological structure of complex microemulsions formed between formulations of anionic surfactants and crude oil. As a matter of fact, regarding chemical EOR processes as surfactant flooding, it is essential to determine the optimal salinity of formulations of surfactants with crude oil in order to get near zero interfacial tensions to mobilize the oil that has been trapped in the porous medium of the reservoir rock. The middle phase microemulsions that form in these Winsor III systems are notably difficult to characterize due to their utmost complexity. In a previous work, we had developed an experimental methodology based on the application of several techniques which was validated in the case of a model microemulsion [19]. A part of this methodology was applied to complex microemulsions of interest, one formed with a representative formulation of anionic surfactants (AI composed of AGES and IOS) and a real crude oil and the other one with the same formulation of surfactants and a model alkane (n-dodecane or C12) which can be considered as the EACN of the crude oil.

First of all, the salinity scans performed with the crude oil system to determine the optimal salinity were particularly uneasy to interpret because the interfaces were difficult to determine by sight, all the phases being colored. To increase the amount of Winsor III middle phase, the amount of surfactant was increased, and the phases appeared as darkcolored. In that case, we demonstrated that MLS can be used to precisely determine the interfaces, which cannot be done by simple visual observation. So the optimal salinity was determined for both systems. The lowest IFT measured by the spinning drop technique was found as expected at this optimum salinity for AI-AL system $\left(2.510^{-3} \mathrm{mN} / \mathrm{m}\right)$, in the same order of magnitude as for AI-C12 system (2.4 $10^{-3} \mathrm{mN} / \mathrm{m}$ ). Another way to confirm the value of the optimal salinity is to measure the evolution of the surfactant concentration in the water phase (by hyamine titration) and of the water concentration in the oil phase (by Karl Fischer titration) all along the salinity scan. We found that both curves cross as expected by the theory at the optimal salinity which corresponds to the transition between Winsor I (o/w microemulsion) and Winsor II (w/o microemulsion) systems. Finally, DSC was used to determine the salinity and the water content of the microemulsions at the optimal salinity, especially with the AI-AL system. It is worth mentioning that the results concerning the water content are consistent with the Karl Fischer method.

The analyses of the structure of the middle phase microemulsions were performed with cryo-SEM/EDS and DSC. An original structure that we called brick wall structure was evidenced by cryo-SEM for both systems (AI$\mathrm{C} 12$ and $\mathrm{AI}-\mathrm{AL}$ ). This structure is different from the bicontinuous one that is generally encountered in Winsor III systems. In fact one phase is surrounded by a layer of another phase, and they seem to make an array. What we call the brick is a smooth phase and the mortar layer, which is brighter, is composed of small particles. The EDS analysis has shown that the smooth phase was essentially composed of water, salt and surfactant, while the mortar layer was made of grains of some hundreds of nm, mainly composed of oil, water, salt and surfactant. The DSC analysis is consistent with what was observed with cryo-SEM and EDS.

So this work allowed to get a better knowledge of complex microemulsions formed between complex formulations of surfactants representative of chemical EOR applications and oil. Furthermore, this study demonstrates that DSC, in addition to classical techniques, appears as an easy and useful technique that can be advantageously used for physico-chemical characterization (determination of 
water content and salinity) as well as for morphological studies. Further studies will now focus on the study of the kinetic of formation of these types of microemulsions, in order to investigate if the structure that has been studied is really the one at equilibrium, or if it tends towards a more stable structure at very long times.

\section{ACKNOWLEDGMENTS}

The authors thank M. Moiré (IFPEN) for helpful discussions.

\section{REFERENCES}

1 Hirasaki G., Miller C.A., Puerto M. (2011) Recent Advances in Surfactant EOR, SPE J. 16, 04, 889-907.

2 Salager J.L., Morgan J.C., Schechter R.S., Wade W.H., Vasquez E. (1979) Mixing rules for optimum phase-behavior formulations of surfactant/oil/water systems, SPE J. 19, 107115.

3 Reed R.L., Healy R.N. (1977) Some physicochemical aspects of microemulsion flooding: a review, in: Shah D.O., Schechter R.S. (eds), Improved Oil Recovery by Surfactant and Polymer Flooding, Academic Press, New York, pp. 383-437.

4 Shinoda K., Friberg S. (1975) Microemulsions: Colloidal aspects, Adv. Colloid Interface Sci. 4, 4, 281-300.

5 Winsor P.A. (1954) Solvent properties of amphiphilic compounds, Butterworths, London.

6 Kaler E.W., Bennett K.E., Davis H.T., Scriven L.E. (1983) Toward understanding microemulsion microstructure: A small angle X-ray scattering study, J. Chem. Phys. 79, 5673-5684.

7 Mittal K.L., Kumar P. (1999) Handbook of microemulsion science and technology, Marcel Dekker, New York.

8 Sripriya R., Muthu Raja K., Santhosh G., Chandrasekaran M., Noel M. (2007) The effect of structure of oil phase, surfactant and co-surfactant on the physicochemical and electrochemical properties of bicontinuous microemulsion, J. Colloid Interface Sci. 314, 712-717.

9 Herzig E.M., White K.A., Schofield A.B., Poon W.C.K., Clegg P.S. (2007) Bicontinuous emulsions stabilized solely by colloidal particles, Nat. Mater. 6, 966-971.

10 Liu H., Wang Y., Lang Y., Yao H., Dong Y., Li S. (2009) Bicontinuous cyclosporin a loaded water-AOT/Tween 85isopropylmyristate microemulsion: structural characterization and dermal pharmacokinetics in vivo, J. Pharm. Sci. 98, 11671176.

11 Saito H., Shinoda K. (1970) The stability of W/O type emulsions as a function of temperature and of the hydrophilic chain length of the emulsifier, J. Colloid Interface Sci 32, 647651.

12 Lindman B., Kamenka N., Kathopoulis T.M., Brun B., Nilsson P.G. (1980) Translational diffusion and solution structure of microemulsions, J. Phys. Chem. 84, 2485-2490.
13 Aveyard R., Binks B.P., Clark S., Mead J. (1986) Interfacial tension minima in oil-water-surfactant systems. Behaviour of alkane-aqueous $\mathrm{NaCl}$ systems containing aerosol OT, J. Chem. Soc. Faraday Trans 1 82, 125-142.

14 Auvray L., Cotton J.P., Ober R., Taupin C. (1984) Concentrated winsor microemulsions: A small angle X-ray scattering study, J. Phys. Fr. 45, 913-928.

15 Teubner M., Strey R. (1987) Origin of the scattering peak in microemulsions, J. Chem. Phys. 87, 3195-3200.

16 Jahn W., Strey R. (1988) Microstructure of microemulsions by freeze fracture electron microscopy, J. Phys. Chem. 92, 8, 2294-2301.

17 Garti N., Aserin A., Tiunova I., Fanun M. (2000) A DSC study of water behavior in water-in-oil microemulsions stabilized by sucrose esters and butanol, Colloids Surf. A: Physicochem. Eng. Asp. 170, 1-18.

18 Peter U., Roux D., Sood A.K. (2001) Observation of a topological relaxation mode in microemulsions, Phys. Rev. Lett. 86, 3340-3343.

19 Fukumoto A., Dalmazzone C., Frot D., Barré L., Noïk C. (2016) Investigation on physical properties and morphologies of microemulsions formed with sodium dodecyl benzenesulfonate, isobutanol, brine, and decane, using several experimental techniques, Energy Fuels 30, 4690-4698.

20 Dalmazzone C., Clausse D. (2001). Microcalorimetry, in: (ed), Encyclopedic Handbook of Emulsion Technology, Marcel Dekker, New York, pp 327-347.

21 Clausse D., Gomez F., Dalmazzone C., Noïk C. (2005) A method for the characterization of emulsions : Thermogranulometry. Application to water-in-crude oil emulsion, $J$. Colloid Interface Sci. 287, 694-703.

22 Clausse D., Gomez F., Pezron I., Komunjer L., Dalmazzone C. (2005). Morphology characterization of emulsions by differential scanning calorimetry, Adv. Colloid Interface Sci. 117, 5974.

23 Dalmazzone C., Noïk C., Clausse D. (2009). Application of DSC for emulsified system characterization, Oil Gas Sci. Technol. Rev. IFP 64, 5, 543-555.

24 Clausse D., Dalmazzone C. (2014) Freezing within emulsions: Theoretical aspects and engineering applications, Oil Gas Sci. Technol. Rev. IFP 69, 3, 415-434.

25 Vonnegut B. (1942) Rotating bubble method for the determination of surface and interfacial tensions, Rev. Sci. Instrum. 13, 6-9.

26 Moiré M. (2015) Etude des propriétés interfaciales eau/huile/ tensioactifs par microfluidique / Study of the interfacial properties of water/oil/surfactants by microfluidics, $\mathrm{PhD}$ Thesis, University Pierre et Marie Curie, UPMC, Paris, France.

27 Issman L., Talmon Y. (2012) Cryo-SEM specimen preparation under controlled temperature and concentration conditions, $J$. Microsc. 246, 60-69.

Manuscript submitted in 31 July 2017 Manuscript accepted in 20 November 2017 Published online in February 2018 